\title{
Mortalidade por causas externas no estado da Bahia, 2015-2019
}

Mortality from external causes in the state of bahia, 2015-2019

Mortalidad por causas externas en el estado de bahia, 2015-2019

Marcela Rossi Ribeiro

ORCID: https://orcid.org/0000-0002-7874-6086 Universidade Estadual do Sudoeste da Bahia, Brasil E-mail: marcelarossiribeiro@gmail.com

Lyra Cândida Calhau Rebouças

ORCID: https://orcid.org/0000-0003-0576-4360 Universidade Estadual do Sudoeste da Bahia, Brasil

E-mail:lyracalhau@gmail.com

Cléber Souza de Jesus

ORCID: https://orcid.org/0000-0001-6727-3247

Universidade Estadual do Sudoeste da Bahia, Brasil

E-mail: csjesus@uesb.edu.br

Roseli Maria Cardoso Ribeiro

ORCID: https://orcid.org/0000-0002-1208-6492

Universidade Estadual do Sudoeste da Bahia, Brasil

E-mail: roseli.cardoso@uesb.edu.br

Anadir de Almeida Farias

ORCID: https://orcid.org/0000-0002-2124-531X

Universidade Estadual do Sudoeste da Bahia, Brasil

E-mail: nnhfarias@gmail.com

Gleide Magali Lemos Pinheiro

ORCID: https://orcid.org/0000-0002-0848-3348

Universidade Estadual do Sudoeste da Bahia, Brasil

E-mail: gleidemlp@gmail.com

Charles Souza Santos

ORCID: https://orcid.org/0000-0001-5071-0359 Universidade Estadual do Sudoeste da Bahia, Brasil

E-mail: charlesss@uesb.edu.br

Maicla Oliveira da Silva

ORCID: https://orcid.org/0000-0002-4536-2262 Universidade Estadual do Sudoeste da Bahia, Brasil

E-mail: maicla64@gmail.com

Maycon Brandão dos Santos

ORCID: https://orcid.org/0000-0002-3012-267X Universidade Federal do Recôncavo da Bahia, Brasil E-mail: maycombrandaos@gmail.com

Rebecca Calheira Barreto

ORCID: https://orcid.org/0000-0001-7555-6714

Universidade Estadual do Sudoeste da Bahia, Brasil

E-mail: rebeccacalheira@gmail.com

Micaela Leão de Sousa

ORCID: https://orcid.org/0000-0002-4726-5837

Universidade Estadual do Sudoeste da Bahia, Brasil

E-mail: micaelaleao_s@hotmail.com

Evelin Matos Marambaia Souza

ORCID: https://orcid.org/0000-0001-9352-5524

Universidade Estadual do Sudoeste da Bahia, Brasil

E-mail: evelinmarambaiaa@gmail.com

Bianca Oliveira Souza Martins

ORCID: https://orcid.org/0000-0002-8939-9630

Universidade Estadual Do Sudoeste da Bahia, Brasil

E-mail: biancamartins9991@gmail.com

Letícia Souza Leal

ORCID: https://orcid.org/0000-0002-3290-0324

Universidade Estadual do Sudoeste da Bahia, Brasil

E-mail: leticiasouzaleal_@hotmail.com

Wicentte Aurélio dos Santos Prates

ORCID: https://orcid.org/0000-0002-9897-983X

Universidade Estadual do Sudoeste da Bahia, Brasil E-mail: conwicentte@gmail.com 


\begin{abstract}
Resumo
Objetivo: descrever o perfil epidemiológico da mortalidade por causas externas no estado da Bahia no período de 2015 a 2019. Metodologia: trata-se de uma pesquisa do tipo descritiva, de delineamento temporal e abordagem quantitativa que utilizou dados secundários do Sistema de Informação de Mortalidade (SIM), do Departamento de Informática do SUS (DATASUS) que são disponibilizados pelo Ministério da Saúde. Resultados: os achados mostraram que entre as causas externas analisadas, a agressão foi a razão das maiores taxas de mortalidade. Ao analisar o perfil das vítimas acometidas pelas causas externas, a maior parte são indivíduos do sexo masculino com idade entre 20 a 29 anos, de cor parda, com escolaridade entre 4 a 7 anos de estudos e a via pública foi o local principal de ocorrência das mortes. Conclusão: constatou-se que no período do estudo a Bahia apresentou uma tendência de aumento de óbitos por causas externas, principalmente no que tange as agressões, um óbito evitável. Acreditamos que com base nos achados apresentados é possível obter subsídios para a elaboração de políticas públicas que visem a promoção da saúde para a população, assim como a redução dos óbitos por causas externas na Bahia e no país.
\end{abstract}

Palavras-chave: Mortalidade; Causas externas; Epidemiologia.

\begin{abstract}
Objective: to describe the epidemiological profile of mortality from external causes in the state of Bahia in the period from 2015 to 2019. Methodology: this is a descriptive study, with a temporal design and a quantitative approach that used secondary data from the Mortality Information System (SIM), from the SUS Department of Informatics (DATASUS), which are made available by the Ministry of Health. Results: the findings showed that among the external causes analyzed, aggression was the reason for the highest mortality rates. When analyzing the profile of victims affected by external causes, most are male individuals aged $\neg 20$ to 29 years, brown, with schooling between 4 to 7 years of schooling and the public road was the main place of occurrence of deaths. Conclusion. it was found that during the study period, Bahia showed a trend towards an increase in deaths from external causes, especially with regard to aggression, a preventable death. We believe that based on the findings presented, it is possible to obtain subsidies for the development of public policies aimed at promoting health for the population, as well as reducing deaths from external causes in Bahia and in the country.
\end{abstract}

Keywords: Mortality; External causes; Epidemiology.

\title{
Resumen
}

Objetivo: describir el perfil epidemiológico de la mortalidad por causas externas en el estado de Bahía en el período de 2015 a 2019. Metodología: se trata de un estudio descriptivo, con un diseño temporal y un enfoque cuantitativo que utilizó datos secundarios del Sistema de Información de Mortalidad. (SIM), del Departamento de Informática del SUS (DATASUS), que puestos a disposición por el Ministerio de Salud. Resultados: los hallazgos mostraron que entre las causas externas analizadas, la agresión fue la causa de las mayores tasas de mortalidad. Al analizar el perfil de las víctimas afectadas por causas externas, la mayoría son individuos del sexo masculino de 20 a 29 años, morenos, con escolaridad entre 4 y 7 años de escolaridad y la vía pública fue el principal lugar de ocurrencia de las muertes. Conclusión. Se encontró que durante el período de estudio, Bahía mostró una tendencia al aumento de las muertes por causas externas, especialmente en lo que respecta a la agresión, una muerte prevenible. Creemos que con base en los hallazgos presentados, es posible obtener subsidios para el desarrollo de políticas públicas orientadas a promover la salud de la población, así como a reducir las muertes por causas externas en Bahía y en el país.

Palabras clave: Mortalidad; Causas externas; Epidemiología.

\section{Introdução}

As causas externas apresentam um notável aspecto na mortalidade em todos os países do mundo, constituindo um número significativo das parcelas de óbitos, especialmente na população masculina brasileira (Messias et al., 2018). A mortalidade é uma variável que se refere ao grupo dos indivíduos que morreram num dado intervalo de tempo. Retrata o risco ou probabilidade que qualquer pessoa apresenta de morrer em decorrência de uma determinada doença ou agravo. Sendo assim, representa a gravidade que os óbitos apresentam numa certa população (Pereira, 2007).

Define-se causas externas como traumatismos, lesões ou quaisquer outros agravos à saúde sejam eles, intencionais ou não que podem ter início súbito como consequência imediata de violência ou outra causa exógena. Neste grupo, incluem-se as lesões provocadas por eventos no transporte, homicídios, agressões, quedas, afogamentos, envenenamentos, suicídios, queimaduras, lesões por deslizamento ou enchente, e outras ocorrências provocadas por circunstâncias ambientais (Gonsaga, et al., 2012). 
A vista disso, no Brasil, nota-se a ascensão crescente na taxa de mortalidade por causas externas nos últimos 25 anos, sendo os óbitos por homicídios e por acidentes de trânsito as principais causas do quadro de mortes violentas. Na mortalidade geral, as causas externas têm uma participação crescente, saindo de 11\% para 15\% no período de 1980 a 2005. Este grupo de causas vem variando entre o segundo e o terceiro lugar de causa de mortalidade, o que confirma a gravidade dessa situação social (Minayo, 2009).

No estado da Bahia, as causas externas representam o segundo grupo de causas de morte mais frequentes e contribuíram com 16\% do total de óbitos registrados em 2009 enquanto, em 2000, este valor foi de 10,6\% (Silva, et al., 2012). Diante do exposto, observa-se que o crescimento da mortalidade por causas externas deve ser encarado como problema de saúde pública.

Assim, a realização desse estudo epidemiológico teve por objetivo: Traçar o perfil epidemiológico da mortalidade por causas externas no estado da Bahia no período de 2015 a 2019, bem como contribuir com as políticas públicas de saúde com o intuito de que a mesma seja capaz de traçar estratégias que visem a prevenção ou diminuição dos óbitos por causas externas.

\section{Metodologia}

Trata-se de uma pesquisa do tipo descritiva, de delineamento temporal e abordagem quantitativa. A pesquisa com análise quantitativa é aquela que se configura pelo emprego de instrumentos estatísticos, tanto na coleta como no tratamento dos dados, e que tem como intenção medir relações entre as variáveis. No estudo quantitativo, por sua vez, o pesquisador parte de um plano preestabelecido com hipóteses e variáveis claramente definidas. Procura medir e quantificar os produtos da investigação, elaborando-os em dados estatísticos (Zanella, 2013).

Nesse caso, a pesquisa descritiva tem como finalidade principal a descrição das características de determinada população ou fenômeno ou, então, o estabelecimento de relações entre variáveis. Diversos estudos podem ser classificados sob este título e uma de suas particularidades mais significativas está na utilização de técnicas padronizadas de coleta de dados, tais como o questionário e a observação sistemática (Gil, 2002). Nessa pesquisa será analisada a mortalidade por causas externas no Estado da Bahia, que tem como população total 14.016.906 pessoas e é composta por 417 cidades. (IBGE, 2010).

Os dados referentes a mortalidade por causas externas foram extraídos do banco de dados do Sistema de Informação de Mortalidade (SIM), do Departamento de Informática do SUS (DATASUS) disponibilizado pelo Ministério da Saúde, dos anos de 2015 a 2019. O ano de 2020 e 2021 não foi incluído no recorte pois não havia informações disponíveis no sistema da coleta que foi realizada entre julho e agosto de 2021. As informações coletadas foram organizadas e analisadas quantitativamente e de modo descritivo através do programa Microsoft Excel. As variáveis utilizadas são referentes a mortalidade por causas externas segundo o local de ocorrência, e estão de acordo com os óbitos decorrentes do Capitulo XX do CID-10 (Causas externas de morbidade e mortalidade), codificados entre V01 e Y98, bem como, idade (1 a 4 anos, 15 a 19 anos, 20 a 29 anos, 30 a 39 anos, 40 a 49 anos, 60 a 69 anos e 80 anos e mais), sexo (masculino e feminino), cor/raça (branca, preta, amarela, parda e indígena) e escolaridade (nenhuma, 1 a 3 anos, 4 a 7 anos, 8 a 11 anos e 12 anos e mais).

Não está sendo analisado os óbitos por residência, que são os ocorridos, contados segundo o local de residência do falecido, as mortes estão sendo analisadas de acordo com a ocorrência, que são os ocorridos, contados segundo o local de ocorrência do óbito. A escolha foi feita pensando em adicionar a variável local de ocorrência e obter um maior detalhamento de acordo com as categorias que a variável apresenta.

Quanto aos aspectos éticos, destaca-se que todos os dados coletados são de domínio público, com acesso irrestrito e disponível pela rede mundial de computadores. A base consultada não contém informações sigilosas, tornando-se abdicada a análise do projeto por um comitê de ética em pesquisa. 


\section{Resultados e Discussão}

A partir da análise dos dados no intervalo de 2015 a 2019 ficou evidente que o total de óbitos na Bahia segundo o grupo CID 10, foi de 66.102 óbitos. Incluído nesse total de óbitos, destaca-se as agressões com um percentual de mortalidade de $52 \%$, em seguida os acidentes de transporte com $20 \%$, os eventos ou fatos cuja intenção é indeterminada com $13 \%$, as quedas com $6 \%$, lesões autoprovocadas intencionalmente com $5 \%$ e por final os afogamento e submersão acidentais com um percentual de 4\%, conforme mostra a Figura 1.

A ocorrência de mortes provocadas por causas externas vem aumentando e provocando grandes alterações no perfil demográfico e epidemiológico da população brasileira, onde se constitui, prioritariamente, não apenas uma questão de saúde, mas também uma questão social (Brasil, 2008).

O estudo de Nogueira e Brandão (2020) aponta que a mortalidade por causas externas traz também consequências para a família, em especial a de jovens. A perda precoce surte efeitos negativos na família, pois os mesmos seriam membros importantes para a colaboração na sustentação econômica, assim como nas relações familiares, como educação dos filhos, isso em indivíduos que já são pais ou são casados. Além disso, esses óbitos ocasionam danos imensuráveis no emocional da família da vítima.

Figura 1: Óbitos por grupo CID 10 na Bahia, no período de 2015 a 2019.

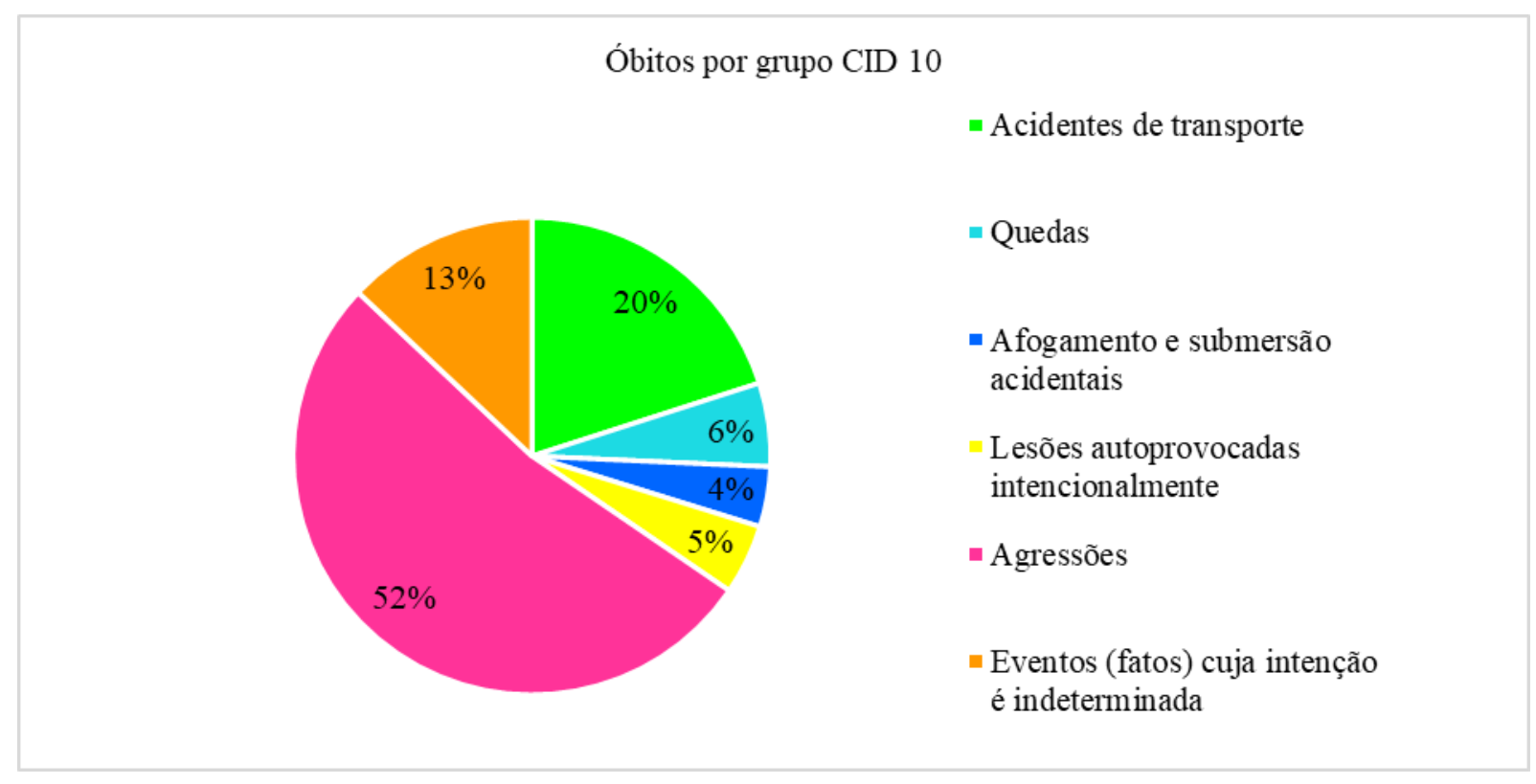

Fonte: MS/SVS/CGIAE - Sistema de Informações sobre Mortalidade - SIM.

Neste estudo, o grupo das agressões, acidentes de transporte e eventos ou fatos cuja intenção é indeterminada ocuparam respectivamente primeiro, segundo e terceiro lugar das causas da mortalidade por causas externas na Bahia. Não é diferente do que acontece no Brasil, de acordo com Marques, et al., (2017), no ano de 2015 foi evidenciado que 145 mil (1.034,46/100 mil habitantes) pessoas foram a óbito vítima das causas externas. Além disso, as agressões e os acidentes de transporte lideram já há alguns anos, variando entre o segundo e terceiro lugar, alguma das maiores razões de mortalidade por causas externas. (Minayo, 2009).

Nas agressões, as mulheres são as principais vítimas de violências domésticas e familiar, chama a atenção esse índice pelo fato da Lei Maria da Penha já existir desde 2006 e ainda hoje, perdurar esse tipo de agressão (Aleixo, et al., 2020). Outro 
fator é a ineficiência e demora de aplicação das medidas de proteção, assim como os profissionais que lidam com essas situações muitas vezes agem com preconceito deixando a mulher vulnerável a agressões por vergonha de denunciar.

Além disso, muitas mulheres não conhecem essa lei nem as medidas de proteção contra a violência que a mesma oferta, como: em relação ao agressor inclui a suspensão da posse ou restrição do porte de armas, o afastamento do lar, e a proibição de aproximação da ofendida, de seus familiares e das testemunhas, fixando o limite mínimo de distância entre estes e o agressor. Em relação a vítima, estão asseguradas o encaminhamento a programa de proteção, a recondução ao domicílio após afastamento do agressor, o afastamento do lar e a separação de corpos, essas são medidas de urgência (Garcia, et al., 2013).

As agressões contra a mulher envolvem um grande número de atos desde a agressão verbal, física, sexual, chegando inclusive ao feminicídio, e os principais agressores são os parceiros ou ex-parceiros (Garcia, et al., 2013). Dessa forma, as mortes de mulheres por agressões são consequências evitáveis, que interrompem de modo precoce as vidas de milhares de brasileiras.

Nos homens, as agressões na maioria das vezes estão relacionadas ao tráfico de drogas, ao uso de drogas ilícitas e a maior acesso a armas de fogo favorecidos pela impunidade (Brasil, 2012). Além disso, segundo Moura, et al., (2015), a cultura relacionada ao gênero estabelece que os homens adotem um modelo de conduta que necessita de agressividade e violência como forma de confirmação da masculinidade, tornando-os mais vulneráveis ao risco de morte.

Em relação aos acidentes de transporte, os maiores índices de mortalidade encontram-se em cidades de médio e pequeno porte, onde a fiscalização não existe ou é pouco efetiva (Minayo, 2009). Validando o exposto, Silva, et al., (2012) aponta que em Jequié, cidade de médio porte do interior da Bahia, os acidentes de transporte tem sido umas das principais causas de mortalidade, representando entre os anos de 2006 a 2010, 79,9\% dos óbitos por causas externas.

Essa situação também ocorre porque a população não obedece às leis de trânsito, com isso fica notório a necessidade de intensificar a educação no trânsito durante a formação dos condutores, assim como, às vezes as vias não possuem uma sinalização necessária, dessa maneira, é preciso que haja estratégias eficazes para uma sinalização suficiente onde os motoristas possam dirigir com segurança e que esse fato seja reconhecido pelas autoridades competentes e pelos responsáveis pelas ações de planejamento no trânsito e na gestão das cidades. Outro fator é o uso abusivo de álcool pelos condutores, essas características se configuram como as principais causas desses elevados números de mortes (Santana, 2021).

Entre esses veículos, as motos possuem uma notoriedade maior devido ao seu aumento crescente e por circularem sem documentações necessárias, tantos dos veículos como das pessoas que o conduzem, além da vulnerabilidade que esse veículo oferece (Minayo, 2009).

No sentido de reduzir a mortalidade por acidentes de transporte algumas medidas vêm surtindo efeito, segundo Preis, et al., (2018), houve uma redução nesses índices depois da implantação da lei seca no ano de 2008, assim como pela exigência do uso do cinto de segurança e da cadeira infantil para as crianças até 10 anos, porém ainda com um efeito discreto sobre a alta prevalência para óbitos por esta causa.

Em todos os anos analisados, os homens apresentam as maiores taxas de morte por causas externas no Estado da Bahia, como mostra na Figura 2. Já no que diz respeito a faixa etária, evidenciou-se que os jovens com idade de 20 a 29 anos do sexo masculino equivalem a um significativo percentual de 37,3\% dos óbitos. 
Figura 2: Taxa de mortalidade por faixa etária e sexo na Bahia, no período de 2015 a 2019.

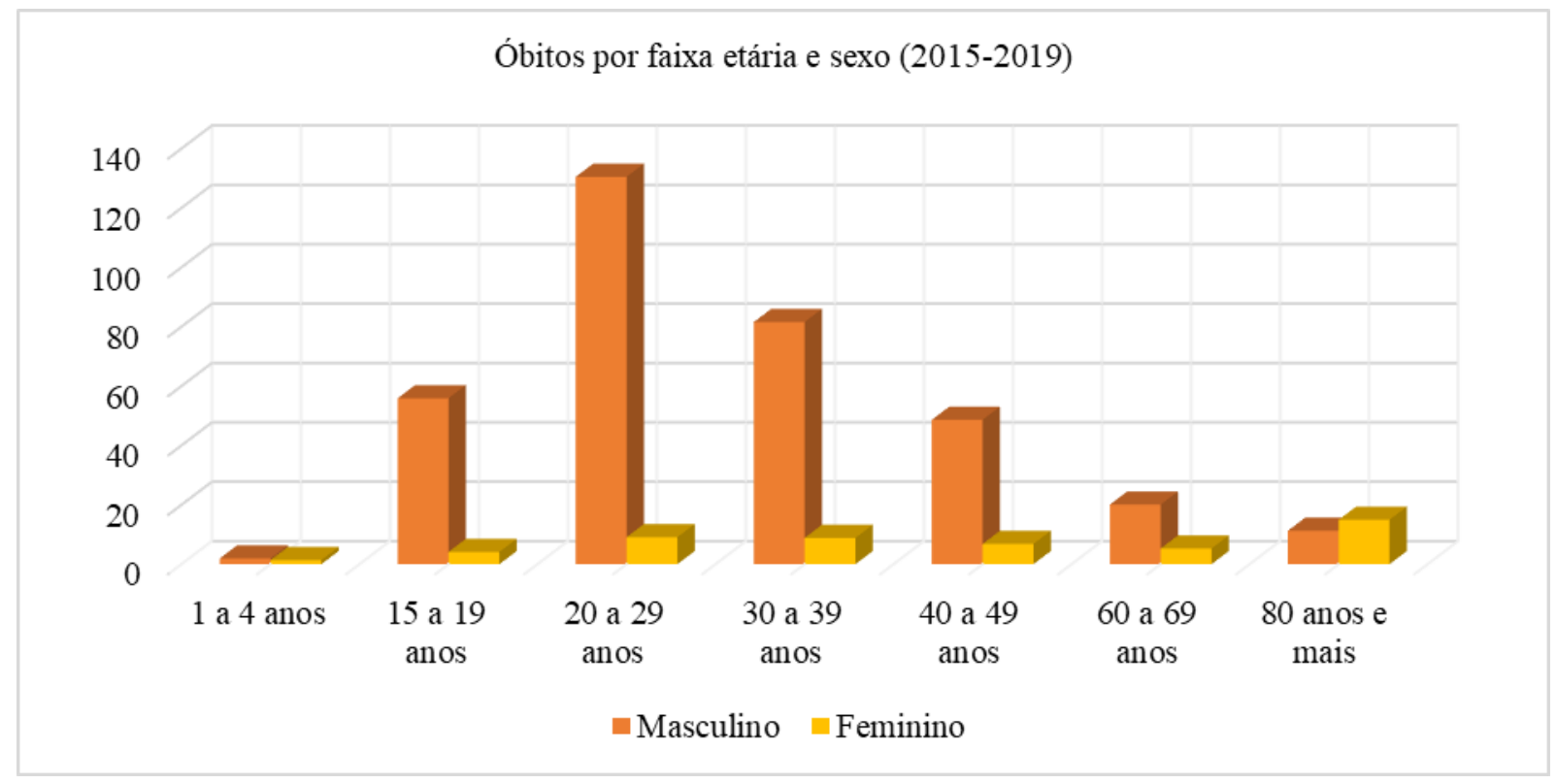

Fonte: MS/SVS/CGIAE - Sistema de Informações sobre Mortalidade - SIM

Essa desigualdade no número de mortes entre os sexos masculino e feminino aqui no Brasil está associada ao comportamento social e cultural, com isso, os homens estão mais sujeitos ao envolvimento em situações de risco, pois esse grupo se envolve com frequência em agressões e discussões, principalmente em brigas no trânsito, consomem grandes quantidades de álcool e dirigem ou pilotam motocicletas em alta velocidade sem uso de cinto de segurança nem capacete, há também o envolvimento em tráficos de drogas e o próprio consumo e o porte de arma de fogo de forma ilegal. Essas condutas aumentam a vulnerabilidade desse grupo (Barros, et al., 2001).

Além de tudo, é importante destacar a faixa etária dos óbitos por causas externas. Nos estudos anteriores de Messias, et al., (2018) e Marques, et al., (2017) também são revelados que são os homens jovens de 20 a 29 anos e de 30 a 39 anos que estão sendo mais acometidos por esse tipo de óbito. Isso aponta que a sensação de liberdade e a falta de maturidade, que são características marcantes na maior parte dos jovens, além da conduta que os mesmos apresentam, como foi citado anteriormente, levam esses homens jovens a morrerem de forma antecipada (Ascari, et al., 2013).

A Figura 3 mostra que em relação a óbitos por cor/raça, constatou-se um percentual de $77 \%$ em pessoas de cor parda, seguido com um percentual de 13\% para indivíduos de cor preta e um percentual de $10 \%$ em pessoas de cor branca.

O dado encontrado corrobora com o estudo de Nepomuceno, et al., (2021) afirmando que a população mais acometida é a de cor não branca assim como, Fiorio, et al., (2011), sustenta que os indivíduos pretos e pardos morrem mais cedo que os brancos, o que mostra que esse índice pode estar relacionado as más condições de vida e ao acesso dificultado e desigual a serviços de saúde para a população negra. Afirma ainda que, para os brancos, o óbito pode acontecer em idades mais avançadas, além de apresentar menor risco de morrer por causas externas o que faz com que esses tenham probabilidade de viver mais tempo e em condições de vida melhor que os negros.

Conclusões iguais a essa foram encontrados por Batista (2005), analisando a mortalidade por raça/cor e sexo no Estado de São Paulo, o mesmo evidenciou que a mortalidade prevalece duas vezes mais em homens negros quando comparado aos brancos. 
Figura 3: Óbitos por cor / raça na Bahia, no período de 2015 a 2019.

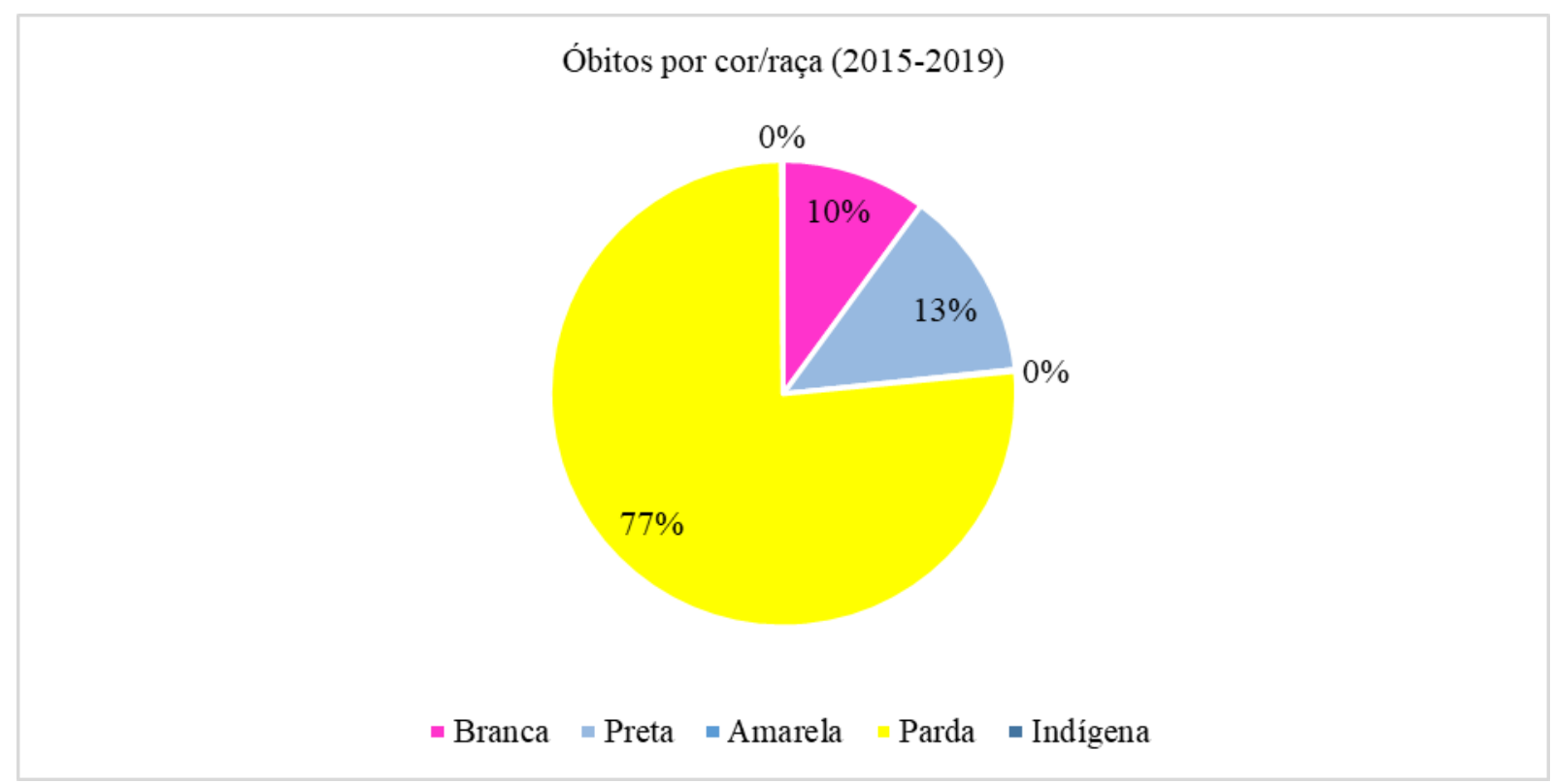

Fonte: MS/SVS/CGIAE - Sistema de Informações sobre Mortalidade - SIM.

A variável escolaridade nesse estudo revelou que 147,91/100 mil habitantes o número de mortes na população de quatro a sete anos de estudos, seguido da escolaridade de 1 a 3 anos com índice de 97,36/100 mil habitantes, como revela a Figura 4.

Figura 4: Óbitos por escolaridade na Bahia, no período de 2015 a 2019.

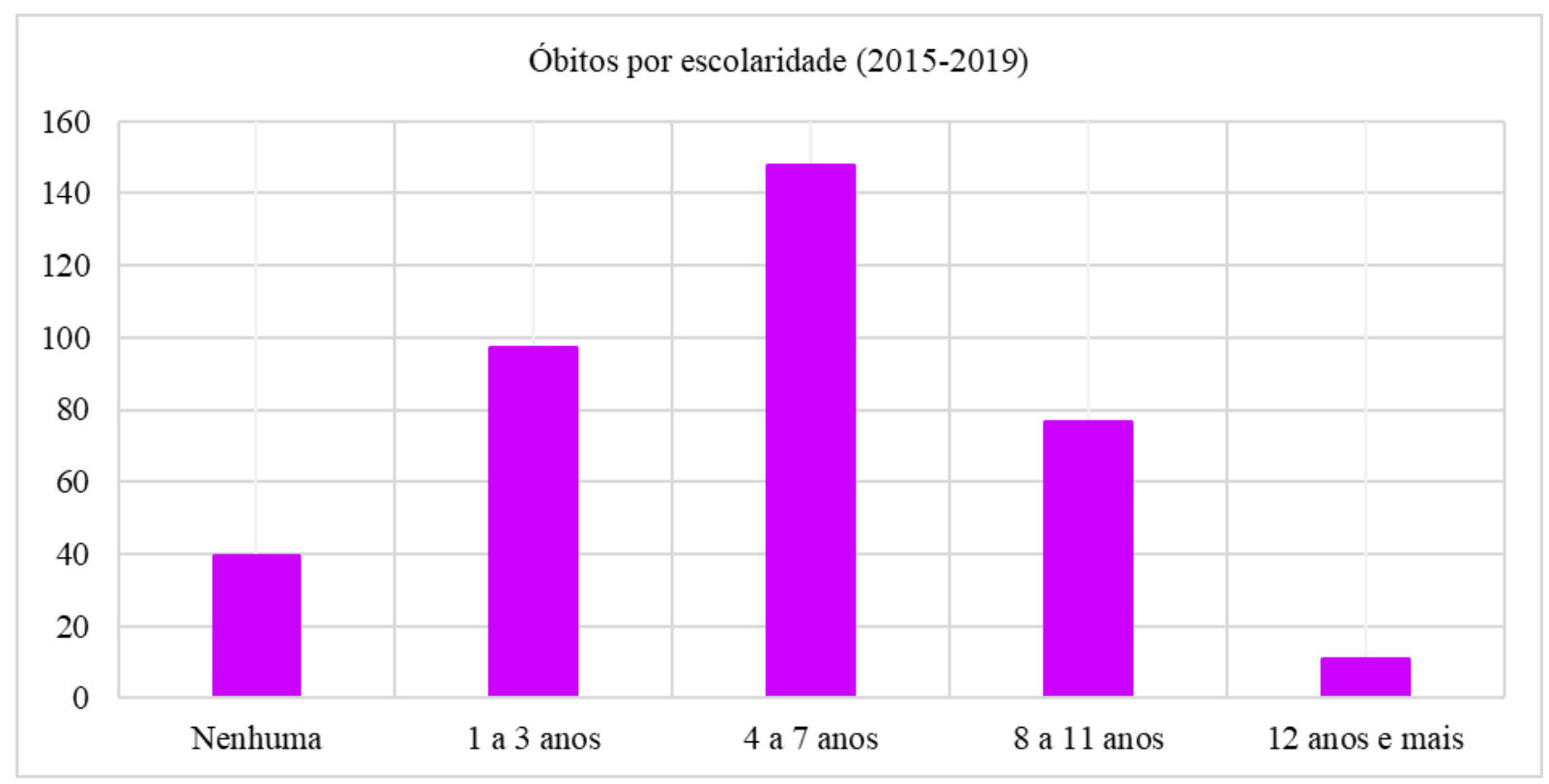

Fonte: MS/SVS/CGIAE - Sistema de Informações sobre Mortalidade - SIM.

Reforça esses dados o estudo de Sousa, et al., (2016), onde mostra resultados semelhantes como o nível de escolaridade muito baixa, afirmando que a maior parte dos óbitos ocorreram com pessoas que estudaram entre 4 a 7 anos, ou seja, não chegaram nem a concluir o ensino fundamental. 
Esse baixo nível de escolaridade afeta de forma negativa o auto cuidado com a saúde, eleva a desigualdade social além de comprometer o desenvolvimento intelectual e até mesmo cultural.

O motivo do abandono dos estudos na maioria das vezes é a busca por emprego para ajudar no sustento da família, essa inserção no mercado de trabalho faz com que o indivíduo abandone o estudo por falta de tempo para conciliar as duas ocupações. A baixa escolaridade leva essas pessoas a uma dificuldade de ascensão social por falta de capacitação, tal fato eleva a vulnerabilidade dessas pessoas levando-as a consequências como a marginalidade e aumentando o risco de morte (Nepomuceno, et al., 2021).

A Figura 5 apresenta os óbitos por causas externas segundo o local de ocorrência, destaca-se a via pública com um percentual de 44\%, o hospital com um percentual de 39\% e o domicílio evidenciando um percentual de 15\% dos óbitos.

Figura 5: Óbitos por local de ocorrência na Bahia, no período de 2015 a 2019.

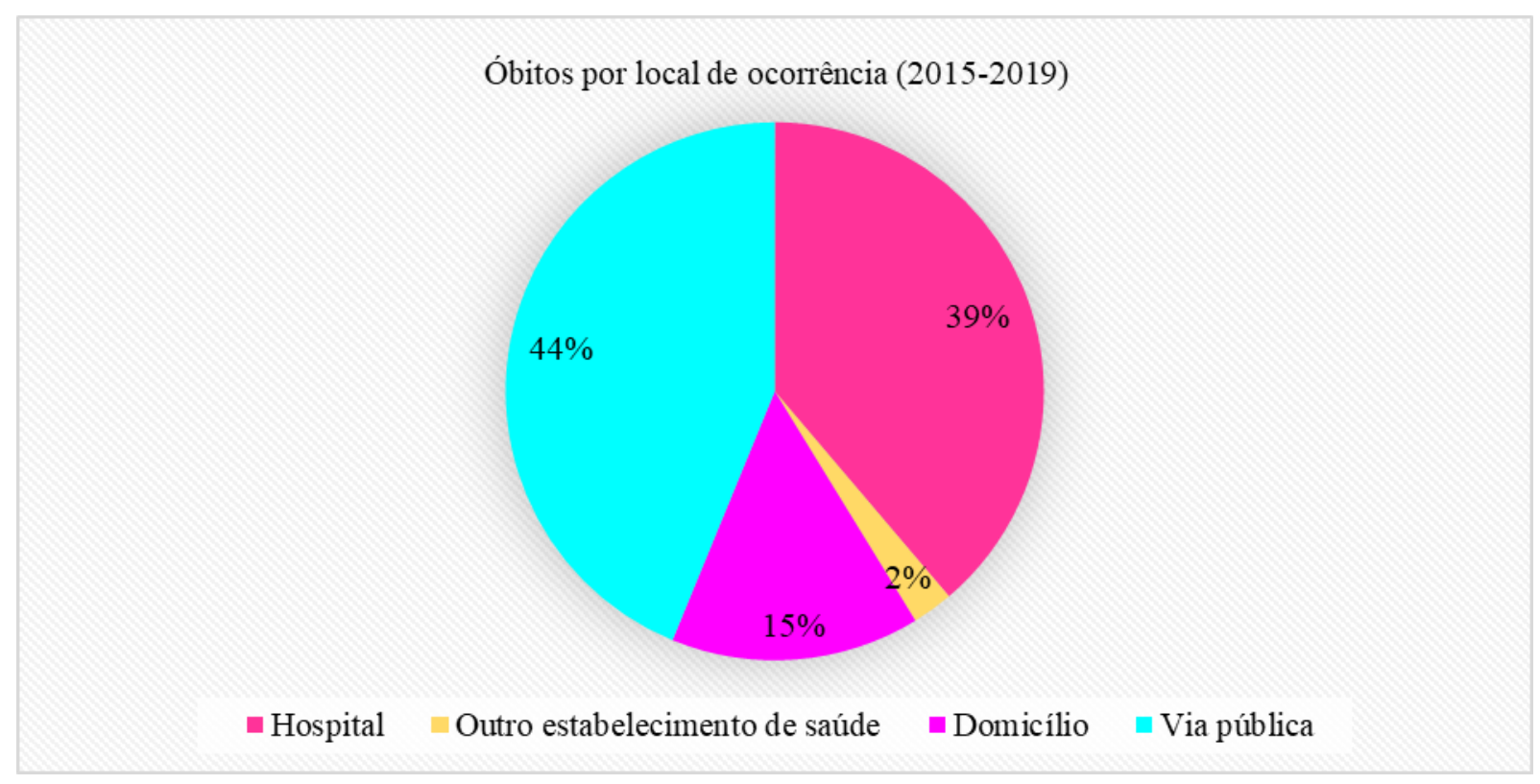

Fonte: MS/SVS/CGIAE - Sistema de Informações sobre Mortalidade - SIM.

O local de ocorrência dos óbitos apontou dados parecidos aos encontrados em uma pesquisa que avalia também o perfil de mortalidade no estado da Bahia, mostrando que a maior parte das mortes ocorreu em via pública, seguido das instituições hospitalares e do domicílio. (Nepomuceno, et al., 2021).

O local de ocorrência onde acontece os maiores índices de mortalidade apresentados acima, são compatíveis com a maior causa de mortalidade, que é a agressão. Há maiores ocorrências de violência envolvendo homens jovens nas vias públicas, diferentemente das mulheres que sofrem com a agressão nos domicílios.

\section{Conclusão}

O estudo demonstra dados inquietantes sobre o número de óbitos por causas externas, principalmente no que diz respeito às vítimas por agressão, com números que se mantém altos e constantes no período de 2015 a 2019 . Outro aspecto que merece destaque é o perfil dessas vítimas, em geral, homens jovens de 20 a 29 anos, não brancos, de baixo grau de escolaridade, vitimados sobretudo nas vias públicas. Isso nos mostra o quanto é necessário que os cidadãos reflitam sobre os modos de comportamento e as atitudes junto a sociedade, obtendo a resolução dos problemas através de diálogo e negociação, rejeitando a violência para solucionar e prevenir conflitos. 
A obtenção de dados secundários através do SIM, apresentou-se como um limite para esse estudo, pela subnotificação dos casos ocorridos, assim como pelo preenchimento incompleto ou incorreto das informações na declaração de óbito. Dessa forma, é importante reconhecer o quanto o registro dos dados, de maneira correta e completa, faz-se importante para a ciência.

É preciso destacar a relevância de estudos que visem a identificação das estatísticas vitais da sociedade, o mesmo fornece subsídios para que o poder público adote medidas de planejamento e implementação de políticas públicas e programas de saúde pública onde visem reduzir a quantidade de óbitos pois, a mortalidade por agressões é evitável, para isso é preciso mesclar ações que promovam uma educação, cultura, cidadania e leis efetivas no combate à violência, a partir de estratégias como essa será possível diminuir o número de vítimas de óbitos por agressões que é muito preocupante na atualidade e tem forte tendência a banalização.

\section{Referências}

Abreu, A. M. M., Jomar, R. T., Thomaz, R. G., Guimarães, F. R. M., Lima, J. M. B., \& Figueirò, R. F. S. (2012). Impacto da Lei Seca na mortalidade por acidentes de trânsito. Revista Enfermagem UERJ, 20(1), 21-26. https://www.e-publicacoes.uerj.br/index.php/enfermagemuerj/article/view/3970/2753

Aleixo, W. F., Graciano, M. B. N., \& Candido T. S. (2020). Mortalidade de mulheres por agressões no Brasil em 2016 E 2017 : um problema de saúde pública subnotificado. (12 ed.). Revista Saúde em Foco, 189-196. https://portal.unisepe.com.br/unifia/wp-content/uploads/sites/10001/2020/06/MORTALIDADE-DEMULHERES-POR-AGRESS\%C3\%95ES-NO-BRASIL-EM-2016-E-2017-UM-PROBLEMA-DE-SA\%C3\%9ADE-P\%C3\%9ABLICASUBNOTIFICADO.docx.pdf

Ascari, R. A., Chapieski, C. M., Silva, O. M., \& Frigo, J. (2013). Perfil epidemiológico de vítimas de acidente de trânsito. Revista de Enfermagem da UFSM, 3(1), 112-121. https://periodicos.ufsm.br/reufsm/article/view/7711/pdf

Barros, M. D.A., Ximenes, R., \& Lima, M. L. C. (2001). Mortalidade por causas externas em crianças e adolescentes: tendências de 1979 a 1995. Revista de Saúde Pública, 35(2), 142-149. https://www.scielo.br/j/rsp/a/FN3RssfBddZPXFQqCMZw8Db/?lang=pt

Batista, L. E. (2005). Masculinidade, raça/cor e saúde. Ciência \& Saúde Coletiva, 10(1), 71-80. https://www.scielo.br/j/csc/a/xcPrrMCTKJvHgswHBWkySyD/?lang=pt

Brasil (2012). Ministério da Saúde. Secretaria de Vigilância em Saúde. Departamento de Análise de Situação de Saúde. Saúde Brasil 2011: uma análise da situação de saúde e a vigilância da saúde da mulher / Ministério da Saúde, Secretaria de Vigilância em Saúde, Departamento de Análise de Situação de Saúde. - Brasília: Ministério da Saúde.

Brasil. (2008). Ministério da Saúde. Informações de saúde demográficas e socioeconômicas. https://datasus.saude.gov.br/demograficas-e-socioeconomicas/

Fiorio, N. M., Flor, L. S., Padilha, M., Castro, D. S. \& Molina, M. C. B. (2011). Mortalidade por raça/cor: evidências de desigualdades sociais em Vitória (ES), Brasil. Rev Bras Epidemiol, 14(3), 522-530. https://www.scielo.br/j/rbepid/a/4hYGKtpqCkpsN45BcgkFHMw/?lang=pt

Garcia, L. P., Freitas, L. R. S. \& Höfelmann, D. A. (2013). Avaliação do impacto da Lei Maria da Penha sobre a mortalidade de mulheres por agressões no Brasil, 2001-2011. Epidemiol. Serv. Saúde, 22(3), 383-394. 10.5123/S1679-4974201300030000

Gil, A. C. 1946 (2002) - Como elaborar projetos de pesquisa/Antônio Carlos Gil. (4ª . ed.). São Paulo: Atlas.

Gonsaga, R. A. T., Rimoli, C. F., Pires, E. A.; Zogheib, F. S.; Fujino, M. V. T.; \& Cunha, M. B. (2012). Avaliação da mortalidade por causas externas. Revista do Colégio Brasileiro de Cirurgiões, 39(4), 263-267. https://www.scielo.br/j/rcbc/a/LHYjWm5Bc68ngyd3PgnmcCb/?lang=pt

IBGE - Instituto Brasileiro de Geografia e Estatística. (2010). Censo Brasileiro de 2010. Disponível em: https: <https://cidades.ibge.gov.br/brasil/ba >. Acesso em: 09 jan. 2021.

Marques, S. H. B., Souza, A. C., Vaz, A. A., Pelegrini, A. H. W., \& Linch, G. F. C. (2017). Mortalidade por causas externas no Brasil de 2004 A 2013. Revista Baiana de Saúde Pública, 41(2), 394-409. 10.22278/2318-2660.2017. v.41.n2.a2368.

Messias, M. M., Bandeira, J. R., Lopes, A. B., Silva, L. L. D., \& Curado, P. F. (2018). Mortalidade por causas externas: revisão dos dados do Sistema de Informação de Mortalidade. Revista da Sociedade Brasileira de Clínica Médica, 16(4), 218-21. https://www.sbcm.org.br/ojs3/index.php/rsbcm/article/view/374/336

Minayo, M. C. S. (2009). Seis características das mortes violentas no Brasil. Revista Brasileira de Estudos da População, 26(1), 135-140. https://www.scielo.br/j/rbepop/a/HKZ36sYffss3fhmyvsmzpQs/?lang=pt

Moura, E. C., Gomes, R., Falcão, M. T. C., Schwarz, E., Neves, A. C. M., \& Santos, W. (2015). Desigualdades de gênero na mortalidade por causas externas no Brasil, 2010. Ciência \& Saúde Coletiva, 20(3), 779-788. 10.1590/1413-81232015203.11172014

Nepomuceno, A. F. S. F.; Figueiredo, M. S.; \& Jesus, V. S. (2021). Perfil de mortalidade por causas externas no estado da Bahia durante o período de 2010 A 2019. Práticas e Cuidado: Revista de Saúde Coletiva, 2(e10975), 1-11. https://revistas.uneb.br/index.php/saudecoletiva/article/view/10975/8384

Nogueira, C. A. S., \& Brandão, F.B. (2020). Mortalidade de adultos jovens por causas externas no Município de Imperatriz - MA, no biênio (2017 - 2018). (9a ed.). Revista Científica Multidisciplinar Núcleo do Conhecimento, 5, 31-58. https://www.nucleodoconhecimento.com.br/saude/mortalidade-de-adultos 
Research, Society and Development, v. 11, n. 2, e17211225675, 2022

(CC BY 4.0) | ISSN 2525-3409 | DOI: http://dx.doi.org/10.33448/rsd-v11i2.25675

Pereira, S. D. (2007). Conceitos e Definições da Saúde e Epidemiologia usados na Vigilância Sanitária.

Preis, L. C., Lessa, Greice., Tourinho, F. S. V., \& Santos, J. L. G. (2018). Epidemiologia da mortalidade por causas externas no período de 2004 A 2013. Revista de Enfermagem UFPE on line, 12(3),716-728. https://periodicos.ufpe.br/revistas/revistaenfermagem/article/view/230886/28032

Santana, J. F. C. L., Xavier, I. F., Zanchetta, V. D., Valentim, F. C. V., Ura, J. F. B., Cestari, C. E., \& Côrtes, M. A. Mortalidade feminina por causas externas em uma região de fronteira: Brasil - Bolívia. (2021). Revista Ciência e Estudos Acadêmicos de Medicina, 4, 78-91. https://periodicos.unemat.br/index.php/revistamedicina/article/view/5448/4290

Silva, J. M., Bispo, K. C. A. B., Andrade, N. M. A. S., Ribeiro, R. M. C. R., Nery, A. A., \& Casotti, C. A. (2012). Mortalidade por causas externas em uma cidade do interior da Bahia. Revista Baiana de Saúde Pública, 36(2), 343-357. https://rbsp.sesab.ba.gov.br/index.php/rbsp/article/view/462/pdf_159

Sousa, A. S. B.; Silva, S. C.; \& Cavalcante, M. F. A. Mortalidade por causas externas em adultos jovens em Teresina-PI no período de 2001-2011. (2016). Revista Interdisciplinar, 9(1),57-65. https://webcache.googleusercontent.com/search?q=cache:kQhjAPTBemEJ:https://dialnet.unirioja.es/descarga/articulo/ 6771955.pdf $+\& \mathrm{~cd}=2 \& \mathrm{hl}=\mathrm{pt}-\mathrm{BR} \& \mathrm{ct}=\mathrm{clnk} \& \mathrm{gl}=\mathrm{br}$

Zanella, L. C. H. (2013). Metodologia de pesquisa / Liane Carly Hermes Zanella (2a ed.). Departamento de Ciências da Administração. 